\title{
Synchronous primary carcinomas of the rectum and prostate: Report of three cases
}

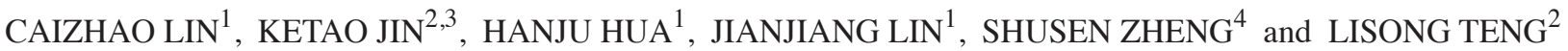 \\ Departments of ${ }^{1}$ Colorectal Surgery and ${ }^{2}$ Surgical Oncology, the First Affiliated Hospital, College of Medicine, \\ Zhejiang University; ${ }^{3}$ Department of Surgery, Affiliated Zhuji Hospital, Wenzhou Medical College; \\ ${ }^{4}$ Key Laboratory of Combined Multi-Organ Transplantation, Ministry of Public Health, the First Affiliated Hospital, \\ College of Medicine, Zhejiang University, Zhejiang 310003, P.R. China
}

Received April 13,2011; Accepted June 2, 2011

DOI: $10.3892 / \mathrm{ol} .2011 .323$

\begin{abstract}
Multiple primary tumors (MPT) are a well-known phenomenon. Rapid advancements in diagnostics and therapeutics have contributed to a significant improvement in the survival rates of cancer patients, and also in an increase in the incidence of cases with multiple primary neoplasms, such as synchronous primary carcinomas of the rectum and prostate. We present a case history of a small number of male patients with synchronous primary carcinomas of the rectum and prostate. Two of the three cases were treated with lower anterior resection (LAR) and radical retropublic prostatectomy (RRP) during the same operation, and 1 case was treated with abdominoperineal resection (APR) and RRP during the same operation. No significant complications occurred during these operations. Our experience with these 3 cases of synchronous primary carcinomas of the rectum and prostate indicated that LAR or APR and RRP can safely be performed in a single operation.
\end{abstract}

\section{Introduction}

Multiple primary tumors (MPT) are a common phenomenon (1). This may be a result of improved clinical awareness, advances in diagnostic technology, possible effects of new environmental carcinogenic factors, and an increased life span of cancer patients that allows them to live long enough to develop a second, third or quadruple primary cancer $(2,3)$.

Correspondence to: Dr L. Teng, Department of Surgical Oncology, the First Affiliated Hospital, College of Medicine, Zhejiang University, 79 Qingchun Road, Hangzhou, Zhejiang 310003, P.R. China

E-mail: 1steng@hos.zju.edu.cn; jinketao2001@zju.edu.cn

Dr S. Zheng, Key Laboratory of Combined Multi-organ Transplantation, Ministry of Public Health, the First Affiliated Hospital, College of Medicine, Zhejiang University, 79 Qingchun Road, Hangzhou, Zhejiang 310003, P.R. China

E-mail: shusenzheng@zju.edu.cn

Key words: multiple primary tumors, prostate cancer, rectal cancer, synchronous primary carcinomas
Rapid advancements in diagnostics and therapeutics have contributed to a significant improvement in survival rates of cancer patients, and also in an increase in the incidence of cases with MPT, such as synchronous primary carcinomas of the rectum and prostate.

We report 3 cases with synchronous primary carcinomas of the rectum and prostate. The diagnosis and surgical management of these cases are described in detail and are discussed further in this report.

\section{Case reports}

Case 1. A 68-year-old Chinese male with an intermittent history of rectal bleeding was admitted to our department. A digital rectal examination (DRE) revealed a tethered, ulcerative, non-circumferential $3-\mathrm{cm}$ mass of the distal to the mid-rectum, right lateral to the posterior anterior midline and located $8 \mathrm{~cm}$ from the anal verge. Colonoscopic biopsy of the rectal mass confirmed a moderately differentiated tubular adenocarcinoma. Laboratory tests, including a differential blood count, transaminases, alkaline phosphatase, bilirubin, albumin, electrolytes, blood urea nitrogen, creatinine and serum carcinoembryonic antigen (CEA) were at normal levels. Serum prostate-specific antigen (PSA) was elevated to $57 \mathrm{ng} / \mathrm{ml}$ (normal range, $0-4 \mathrm{ng} / \mathrm{ml}$ ). The pelvic magnetic resonance imaging (MRI) revealed rectal masses and space-occupying lesions in the prostate (Fig. 1). Transrectal ultrasound (TRUS)-guided biopsy of the prostate revealed an adenocarcinoma (the right lobe of the prostate).

Lower anterior resection (LAR) and radical retropublic prostatectomy (RRP) were performed in a single operation. Surgery consisted of an initial pelvic lymphadenectomy and non-nerve-sparing RRP in a standard manner through a lower midline incision. Prior to the completion of the bladder anastomosis, bilateral single-J urinary diversion stents were passed up into each renal pelvis. The distal ends were brought out through the anterior bladder wall and the anterior abdominal wall. The LAR of the rectum was then performed, with a primary, hand-sewn, end-to-end proctosigmoidostomy. The fecal stream was diverted with a transverse loop colostomy.

Postoperative pathological sections showed two morphologically distinct primary tumors, and the patient was 


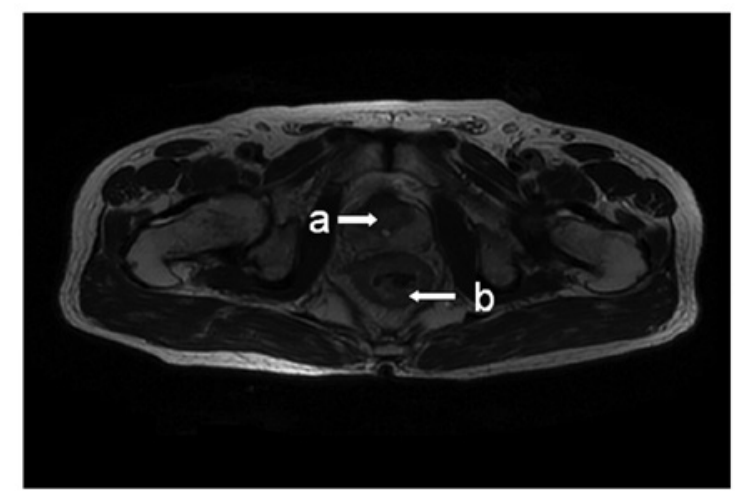

Figure 1. Pelvic magnetic resonance imaging (MRI) showing an isolated weak signal nodule in the (a) peripheral zone and a swelling mass in the posterior wall of the distal rectum, involving (b) the serosa.

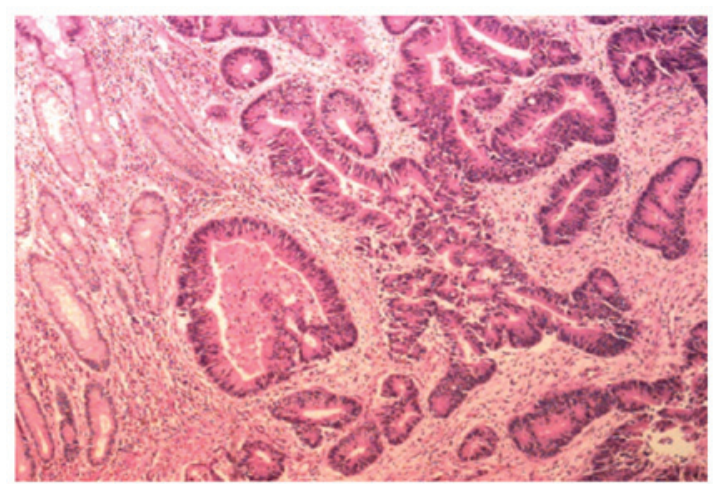

Figure 2. Histological section showing moderately differentiated rectal tubular adenocarcinoma. Microscopic examination revealed a distinct adenic structure in the cancer nest (H\&E staining; magnification, x100).

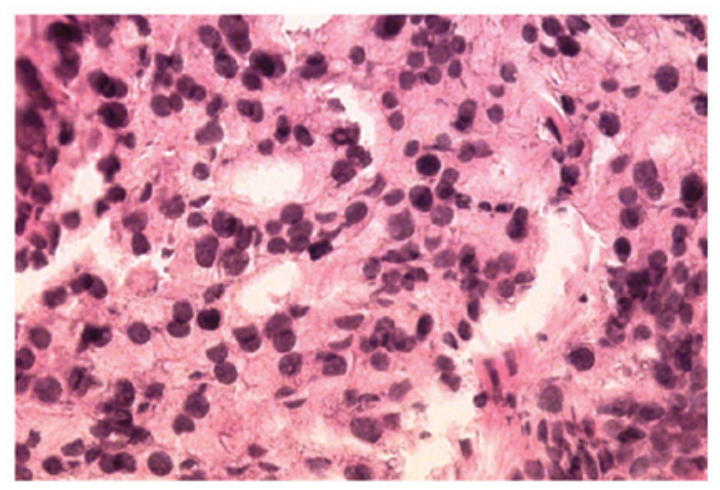

Figure 3. Histological section showing undifferentiated adenocarcinoma of prostate. Microscopic examination revealed small tumor cells, lined up into a solid mass, and a lack of adenic structure (H\&E staining; magnification, $\mathrm{x} 400$ ).

diagnosed with two synchronous pelvic malignancies: a T4NOM0 adenocarcinoma (Dukes' stage B) of the rectum (Fig. 2) and a $\mathrm{T} 2 \mathrm{~N} 2 \mathrm{M} 0$ adenocarcinoma [Gleason grade $5(3+2)$ ] of the prostate (Fig. 3). Subsequently, the patient was primarily administered FOLFOX4 regimen (leucovorin, 5-fluorouracil and oxaliplatin) as adjuvant chemotherapy. After 4 cycles, the patient was switched to FORFILI regimen (irinotecan, leucovorin and 5-fluorouracil). When non-tolerated diarrhea was observed after 2 cycles of FORFILI therapy, the patient was switched to capecitabine (Xeloda, Roche, USA) orally for 2 weeks, followed by a 1-week rest period (3-week cycle) for 4 cycles. The patient succumbed, with recurrence, after 47 months of regular follow-up after surgery.

Case 2. A 65-year-old Chinese male with a history of six months of rectal bleeding was admitted to our department. DRE revealed a tough, ulcerative, non-circumferential $3-\mathrm{cm}$ mass of the distal to mid-rectum, right lateral to the anterior midline and located $4 \mathrm{~cm}$ from the anal verge. Colonoscopic biopsy of the rectal mass confirmed a poorly differentiated tubular adenocarcinoma. Laboratory tests showed an elevated serum PSA (27 ng/ml), but differential blood count, transaminases, alkaline phosphatase, bilirubin, albumin, electrolytes, blood urea nitrogen, creatinine and serum CEA were at normal levels. A pelvic MRI revealed rectal masses and space-occupying lesions in the prostate (data not shown). TRUS-guided biopsy of the prostate revealed an adenocarcinoma (the right lobe of the prostate).

LAR and RRP were performed in a single operation. Surgery was performed in the same manner as described for case 1 .

Postoperative pathological sections showed two morphologically distinct primary tumors, and the patient was diagnosed with two synchronous pelvic malignancies: a T4N1M0 adenocarcinoma (Dukes' stage C) of the rectum and a T3N0M0 adenocarcinoma [Gleason grade $4(2+2)$ ] of the prostate (data not shown). The patient received FOLFOX4 regimen for 12 cycles. After 20 months of regular follow-up, the patient remains asymptomatic.

Case 3. A 70-year-old Chinese male with an intermittent history of rectal bleeding was admitted to our department. DRE revealed a tethered, ulcerative, non-circumferential mass of the distal to mid-rectum, left lateral to the anterior midline and located $4 \mathrm{~cm}$ from the anal verge. Colonoscopic biopsy of the rectal mass confirmed a poorly differentiated tubular adenocarcinoma. Laboratory tests showed a markedly elevated serum PSA (65 ng/ml). A pelvic MRI revealed rectal masses and space-occupying lesions in the prostate (data not shown). TRUS-guided biopsy of the prostate revealed an adenocarcinoma (the left lobe of the prostate).

Abdominoperineal resection (APR) and RRP were performed in a single operation. The surgery started with the intraperitoneal mobilization of the sigmoid colon and rectum as far distally as possible, followed by division of the bowel at the junction of the sigmoid colon with the rectum. The preperitoneal space was then developed to allow the bilateral pelvic lymphadenectomy and RRP in a standard retropubic manner. The perineal dissection and completion of the rectal resection were performed prior to the bladder anastomosis. The levator ani muscles were approximated with 0 chromic suture to provide posterior support to the urethra. Following completion of the bladder anastomosis, the peritoneum was approximated to cover the rectal defect. In an effort to prevent the bladder from falling back into the rectal fossa, several 2-0 chromic sutures were used to secure the anterior bladder wall to the posterior side of the symphysis pubis. The sigmoid colostomy was brought out and matured in the left lower abdomen.

Postoperative pathological sections showed two morphologically distinct primary tumors, and the patient was diagnosed 
with two synchronous pelvic malignancies: a T3NOM0 adenocarcinoma (Dukes' stage B) of the rectum and a T3N1M0 adenocarcinoma [Gleason grade $5(2+3)$ ] of the prostate (data not shown). The patient was received FOLFOX4 regimen for 12 cycles. The post-operative hospitalization time period of the patient was 16 days. After 24 months of regular follow-up, the patient remains asymptomatic.

\section{Discussion}

Synchronous primary carcinomas of the rectum and prostate are rare. DRE and colonoscopy are two relatively convenient methods of detecting rectal cancer (4). The mass location and whether it is mobile or fixed was evaluated to determine the resectability and select an appropriate surgical procedure. Using MRI and a transanal ultrasound, clinicians are able to characterize the tumor and identify tumor draining lymph nodes and distant metastasis.

However, the diagnostic value of DRE in primary care for un-palpable prostatic tumor is limited. Prostate cancer can be diagnosed early in the natural history of the disease by the detection of an elevated serum PSA, before the tumor is palpable on examination or causes symptoms. Although PSA, combined with DRE, provided higher specificity for prostate cancer screening (5), the elevation of PSA beyond the normal range may include benign prostate hyperplasia, old age, prostate trauma and prostate cancer (6). Clinicians should be aware of the possibility of synchronous primary rectal and prostate carcinomas as the delay in diagnosis of the second primary tumor was frequently the result of omission and poor awareness of multiple primary tumors (7). Although $75 \%$ of elderly males with a raised PSA received benign biopsy results (8), transanal ultrasound-guided prostate needle biopsy proved to be essential for a definitive diagnosis of multiple primary tumors, including both those in the rectum and prostate, if an elevated PSA level is detected in rectal cancer patient serum. In our patients, whose rectal carcinoma was detected first, the elevated PSA suggested the possibility of synchronous primary carcinomas of the rectum and prostate, which were confirmed by a subsequent biopsy and postoperative pathological sections of prostate. In our patients, it was possible that direct invasion of the primary rectal carcinoma into the prostate had freed PSA from the prostate tissue and thus elevated serum PSA levels. However, MRI and a direct comparison of pathological sections did not support this diagnosis.

The presence of an incidental primary malignancy in particular often changes patient management. When a patient with synchronous primary malignancies of the rectum and prostate was found, the recommended treatment was LAR or APR of the rectum and RRP. Published reports showed that simultaneous LAR or APR of the rectum and RRP may be performed safely $(9,10)$. In our small series of male patients with synchronous primary carcinomas of the rectum and prostate, 2 patients were treated with LAR and RRP in the same operation, and 1 case was treated with APR and RRP in the same operation. No significant complications occurred in these patients. Results from the three patients suggested the morbidity from the combined procedure to be low.

The risk of fistula formation between the bowel and bladder due to the presence of adjacent, overlying anastomoses is our major concern with combined RRP and LAR of the rectum. To minimize this risk, temporary diversion of the urinary and fecal streams should be attempted. Avoidance of any tension on either anastomosis, or the placement of an adjacent drain, and positioning of a pedicle of omentum between the bowel and bladder should also be attempted to minimize this risk. The risk of urinary incontinence is our major concern with regard to performing RRP with rectal APR. Such a risk can be minimized by putting the levators in near proximity behind the urethra after completion of the perineal resection.

In conclusion, to the best of our knowledge, these are the first three Chinese males with synchronous primary carcinomas of the rectum and prostate to be reported in the English literature. Our experience with the three cases of synchronous primary carcinomas of the rectum and prostate suggested that clinicians should be aware of the possibility of synchronous primary rectal and prostate carcinomas when an elevated PSA is detected in a patient with rectal carcinoma. Our findings also indicated that LAR or APR and RRP may safely be performed in a single operation.

\section{Acknowledgements}

This study was supported by the State Key Basic Research and Development Program of China (973 Program, Grant No. 2009CB521704), National High-tech Research and Development Program of China (863 Program, Grant No. 2006AA02A245), Zhejiang Provincial Science and Technology Project (Grant No. 2009C13021), and Zhejiang Provincial Science Research Foundation for Returned Overseas Chinese Scholars (No. J20100113).

\section{References}

1. Merimsky O, Kollender Y, Issakov J, Bickels J, Flusser G, Gutman M, Lev-Chelouche D, Inbar M and Meller I: Multiple primary malignancies in association with soft tissue sarcomas. Cancer 91: 1363-1371, 2001.

2. Mussari S, Amichetti M and Tomio L: Quadruple cancer in a single patient: a report of four cases. Eur J Surg Oncol 26: 614-616, 2000.

3. Angurana SL, Kapoor R, Kumar P, Khosla D, Kumar N, Sharma SC and Patel FD: Quadruple malignancy in a single patient: a case report and comprehensive review of literature. J Cancer Res Ther 6: 230-232, 2010.

4. Ang CW, Dawson R, Hall C and Farmer M: The diagnostic value of digital rectal examination in primary care for palpable rectal tumour. Colorectal Dis 10: 789-792, 2008.

5. Catalona WJ and Loeb S: Prostate cancer screening and determining the appropriate prostate-specific antigen cutoff values. J Natl Compr Canc Netw 8: 265-270, 2010.

6. Stenman UH, Leinonen J, Zhang WM and Finne P: Prostatespecific antigen. Semin Cancer Biol 9: 83-93, 1999.

7. Yamamoto S, Yoshimura K, Ri S, Fujita S, Akasu $\mathrm{T}$ and Moriya Y: The risk of multiple primary malignancies with colorectal carcinoma. Dis Colon Rectum 49: S30-S36, 2006.

8. Macefield RC, Metcalfe C, Lane JA, Donovan JL, Avery KN, Blazeby JM, Down L, Neal DE, Hamdy FC, Vedhara K and ProtecT Study Group: Impact of prostate cancer testing: an evaluation of the emotional consequences of a negative biopsy result. Br J Cancer 102: 1335-1340, 2010.

9. Campbell SC, Church JM, Fazio VW, Klein EA and Pontes JE: Combined radical retropubic prostatectomy and proctosigmoidectomy for en bloc removal of locally invasive carcinoma of the rectum. Surg Gynecol Obstet 176: 605-608, 1993.

10. Baur H, Frimberger $M$ and Altwein JE: Simultaneous radical prostatectomy and partial rectum resection without colostomy. Eur Urol 31: 380-381, 1997. 